J. Gynäkol. Endokrinol. AT 2021 · 31:136-138 https://doi.org/10.1007/s41974-021-00204-9 Angenommen: 18. Oktober 2021 Online publiziert: 9 . November 2021

๑ Der/die Autor(en) 2021

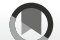

Es dauert meist Jahre, bis eine bei Gericht eingebrachte Klage zu einem Vorwurf medizinischen Fehlverhaltens ausjudiziert ist. Dies vor allem dann, wenn die nächste Instanz bemüht wird und das Verfahren bis zum Obersten Gerichtshof (OGH) gelangt. Wenn dieser es dann an die erste Instanz zurückverweist, können zwischen dem strittigen Ereignis und dem abschließenden Urteil auch 15 Jahre vergehen. Dieses Urteil/diese Erkenntnis wird dann genau studiert, kommentiert und mit mahnendem Unterton zitiert. Nur inzwischen hat sich die Medizin weiterentwickelt, die Situation, wie sie vor 15 oder mehr Jahren zum Klagsfall führte, würde sich heute nicht mehr so stellen, weil sich Labor, Ultraschall und Aufklärungskultur anders entwickelt haben.

Und so werden die Erkenntnisse von Höchstgerichten und einzelne Zitate daraus zu einem Fundus für Analogieschlüsse und Metaphern, die man auf heutige Sachverhalte und Fragestellungen stülpt. Diese Leidenschaft für die Normfindung mittels Exegese weiser alter Texte und ihre spekulative Anwendung auf die Jetzt-Zeit war und ist ein Kennzeichen der Theologie, die Medizin hatte dieses Denken eigentlich im Lauf des 18. Jahrhunderts hinter sich gelassen.

Allerdings kommen diese archaisch-prämodernen Denkmuster wieder zurück, wenn darüber nachgedacht wird, ob ein in den 1950er-Jahren geprägter Leitsatz zum Thoraxröntgen beim Tuberkulosescreening auch für die Metastasensuche

\title{
Christoph Brezinka
}

Department Frauenheilkunde, Universitätsklinik für Gynäkologie und Geburtshilfe, Medizinische Universität Innsbruck, Innsbruck, Österreich

\section{Was ändert sich bei Beschwerden und Klagen zu Gynäkologie?}

mittels PET-CT Gültigkeit haben könnte. Tatsache ist, dass Fälle erst ab einem bestimmten Niveau im Rechtsstreit - irgendwo zwischen Oberlandesgerichten und OGH - überhaupt wahrgenommen und kommentiert werden. Vereinzelt gelangen auch vor Patientenanwaltschaften und Schlichtungsstellen behandelte „Erregungsfälle“ (Kopetzki) an das Licht der Öffentlichkeit, tauchen aber in Ermangelung einer Publikation allfälliger Schlichtungsergebnisse rasch wieder unter.

Man kann viel Zeit und Schaffenskraft damit verbrauchen, zu raten, wie der OGH heute einen bestimmten Sachverhalt nach einem verwinkelten Instanzenzug beurteilen würde, und kann versuchen, diese Mutmaßungen auf einer gezielten Anamnese und Exegese aller Erkenntnisse seit $1950 \mathrm{zu}$ basieren. Oder man kann sich die Fälle, die jetzt in Schlichtungsstellen, Patientenanwaltschaften und Erstgerichten eingebracht werden, anschauen und daraus auf die weitere Entwicklung der Rechtsprechung schließen. Diese wird in der Medizin vor allem dadurch getrieben, was von Betroffenen als Schaden, Fehler und insgesamt als Unrecht wahrgenommen und zur Entscheidung vorgelegt wird. Sehen wir uns die derzeit aktuellen Themenbereiche an:

\section{Endometriose}

Das Klags-/Beschwerdemuster umfasst hier in erster Linie den Vorwurf der Diagnoseverzögerung sowie die fehlende Einschätzung der Endometriose als das Krankheitsgeschehen und das Leben der Patientin dominierenden Prozess: Letzteres etwa, wenn es bei einer tief infiltrierenden Endometrio- se mit Adenomyose unter den Wehen $\mathrm{zu}$ einer Uterusruptur kommt. Klagen wegen Diagnoseverzögerung gibt es in allen Bereichen der Onkologie schon seit Langem, etwa wegen Defiziten bei der Durchführung des Pap-Screenings. Die Endometriose weist eine Vielzahl von Symptomen auf, von vielen Frauen werden diese als Teil des Frau-Seins wahrgenommen, viele Ärzte bestätigen sie darin. Das liegt auch daran, dass die Ausdehnung des Befalls mit Endometriose in keiner unmittelbaren Korrelation mit der Schmerzsymptomatik der betroffenen Patientinnen steht. Über viele Jahre wurde die Diagnose einer Endometriose erst gestellt, wenn die Patientin wegen unerfülltem Kinderwunsch laparoskopiert wurde. In der bisher größten internationalen Vergleichsstudie "Global Study on Women's Health" (GSWH) zur Auswirkung der Endometriose auf Arbeitsproduktivität und Lebensqualität wurde eine durchschnittliche Diagnoseverzögerung von $6 \frac{1}{2}$ Jahren errechnet [1]. Die derzeit zunehmenden Beschwerden bei Schlichtungsstellen sowie die Klagen bei Gericht wegen vorgeworfenen Fehlern bei Diagnose und Therapie von Endometriose werden von den Sachverständigen gute Kenntnisse der neueren Entwicklungen von Diagnostik und Therapie dieses komplexen Krankheitsbilds verlangen. Die Endometriose ist in den letzten Jahren in der Medizin als komplexe chronische Erkrankung anerkannt worden [2]: Sie wird in den Verfahren vor österreichischen Arbeitsund Sozialgerichten um Berufsunfähigkeitspension und Reha-Geld einen größeren Stellenwert bekommen. In Italien haben mehrere Regionen eigene Gesetze verabschiedet, die die „soziale 
Relevanz" dieser Erkrankung unterstreichen sollen [3]. Die Endometriose wird im ärztlichen Bewusstsein von ihrer traditionellen Rolle als letzter Punkt einer langen Liste von Ausschlussdiagnosen weiter nach oben rücken, Patientinnen werden zunehmend danach fragen. Ausführliche Diagnostik und Aufklärung zu den Therapieoptionen sollten in zertifizierten Endometriosezentren unter konsequenter Anwendung der aktuellen Klassifikationen erfolgen [4].

\section{Verhütung - Pille und Spiralen}

Die deutsche Innenpolitik und vor allem die Personalsituation der CDU sähe heute anders aus, wäre der vielversprechende Hoffnungsträger Peter Mißfelder nicht 2015 im Alter von 35 Jahren an einer Lungenembolie verstorben. Bei einer im gleichen Alter mit dieser Diagnose verstorbenen Frau wäre sicher die hormonelle Verhütung angeschuldigt worden, die sie gerade, oder auch in den Jahren zuvor, verschrieben bekommen hatte und es hätte wieder eine Pillenpanik gegeben.

Die leichte Erhöhung der Thrombosegefahr bei der Einnahme von oralen Kontrazeptiva (OC) ist seit Ende der 1960er-Jahr bekannt. Die Möglichkeiten von APC-Resistenztests (Faktor V-Leiden) stehen heute vor dem Verschreiben von $\mathrm{OC}$ ohne nennenswerte Barrieren der Versicherungen problemlos zur Verfügung. Hohe Forderungen an das Niveau und die Dokumentation der Aufklärung über Thromboserisiken vor der Pillenverschreibung sollten spätestens seit einer Erkenntnis des OLG Graz (8C 201/07i) aus dem Jahr 2008 allen Verhütungsverschreibern bekannt sein. Hierbei geht es darum, die Patientin auf thrombogene Situationen und thrombosefördernde eigene Verhaltensweisen aufmerksam zu machen. Die Zeiten der freihändigen Vergabe von Pillenmustern und der Rezepterneuerungen durch die Sprechstundenhilfe sind vorbei.

Klagen und Beschwerden im Zusammenhang mit Spiralen („intrauterine device“ [IUD]) gibt es seit Jahren und sie betreffen meist Uterusperforationen oder den Verlust des IUD sowie ungewollte Schwangerschaften bei liegendem oder verlorenem IUD. Bei der Bewertung wird die Dokumentation der Aufklärung vor dem Legen der Spirale eine große Rolle spielen, weiters die Ultraschalldokumentation eines adäquaten Sitzes des IUD nach dem Legen und bei der obligaten Nachkontrolle einige Wochen später. Von nicht zu unterschätzender Wichtigkeit ist die ärztliche Dokumentation der Produktions-/Seriennummer des IUD, das Vorhandensein eines CE-Siegels des Produkts (und damit die Zulassung im EU-Raum) und die Sicherstellung der Lieferkette. Dies vor allem dann, wenn die IUD von den Patientinnen nicht in der Apotheke gekauft werden, sondern direkt über die Praxis vertrieben werden. Immer wieder kommt es vor, dass Frauen beim Urlaub im Ausland günstig erworbene Spiralen eingesetzt bekommen wollen. Das IUD ist aber ein Implantat, ähnlich einem Herzschrittmacher, weshalb die Gynäkologin eine zu große Verantwortung auf sich nehmen würde - vor allem, wenn es sich um einen in einer asiatischen Apotheke erworbenen „Hosentaschenimport" mit unklarer Provenienz handelt.

Spiralen, die intrauterin zerbrechen und/oder unvollständig entfernt werden, waren bisher eine Rarität. Ein spanischer Hersteller, der mit einer offenbar aus minderwertigem Plastik gefertigten Spirale auf den deutschen und österreichischen Markt drängte, sorgte dafür, dass es zu einer Häufung solcher Vorfälle kam, begleitet vom Medienecho, Gerichtsverfahren und Sammelklagen [5]. Die Entwicklung der Sammelklagen und der einzelnen Klagsvorbringen sowie ihre mediale Präsentation wird in den nächsten Jahren zeigen, ob diese Materialfehler das Image der Spiralen als Verhütungsmittel bei den Verbraucherinnen/Patientinnen nachhaltig schädigen werden.

\section{Niveauabsturz vom geburtshilf- lichen zum gynäkologischen Ultraschall}

Der Ultraschall an der Schwangeren - vom Ersttrimesterscreening über das Organscreening bis zum Doppler im letzten Trimenon - hat in den letzten Jahren in Österreich ein hohes Niveau und festgelegte Standards erreicht. Der gynäkologische Ultraschall ist aber - so wie der Schwangerenschall vor 30 Jahren - noch geprägt durch chaotische Autodidaktik, wo die jüngste Assistentin von der zweitjüngsten den Unterschied zwischen Uterus und Ovar lernt und sich dann entlang einer selbstdefinierten Lernkurve diagnostisch weiterhangelt. Dabei ist die Qualität der Bildgebung an sich durch die Verfügbarkeit hochauflösender Vaginalsonden deutlich besser geworden. Besondere Defizite gibt es auch in der Dokumentation - niemand käme heute auf die Idee, das Ergebnis einer NT-Messung an den Rand einer Ambulanzkarte zu kritzeln und einen unbeschrifteten Thermoprinter-Ausdruck ohne Patientinnennamen an den Rand zu tackern. An vielen Abteilungen und Ordinationen ist dies aber für gynäkologische Ultraschalluntersuchungen die dokumentatorische Alltagspraxis und Vorschläge zur Verbesserung - etwa die Eingabe der Untersuchungsergebnisse und der Fotos in die ohnehin schon für die Schwangerschaft verwendeten Dokumentationsprogramme, die durchwegs auch einen Gyn-Modus haben - werden als Zumutung empfunden. Wenn dann der gynäkologische Ultraschall in erster Linie als Wünschelrute dient, um viele Operationsindikationen zu finden, und wenn nach Komplikationen bei der Operation blander Zysten bei Gericht die Frage nach der Sinnhaftigkeit der Operation gestellt wird, wird aus den Dokumentationsdefiziten rasch ein Argumentationsnotstand.

Die seit über 20 Jahren erarbeiteten IOTA-Kriterien sollten für den gynäkologischen Ultraschall ähnlich verbindlich sein wie die für den Schwangerenultraschall selbstverständlich akzeptierten Standards von FMF und ÖGUM/DEGUM [6]. Auch hier wird man nicht an Kursen und Zertifizierungen vorbeikommen, die im Bereich des Schwangerenultraschalls schon lange akzeptiert werden [7]. Hier schließt sich auch der Kreis zu den Punkten 1 (Endometriose) und 2 (Spiralen) - nur mit adäquatem Ultraschall, einigermaßen standardisierter Befundsprache und Befunddokumentation wird man den heute bestehenden Anforderungen genügen können. 


\section{Intimchirurgie}

Der Tatbestand der weiblichen Genitalverstümmelung ist in Österreich im neu gefassten $₫ 90$ Abs. 3 des StGB geregelt:

3) In eine Verstümmelung oder sonstige Verletzung der Genitalien, die geeignet ist, eine nachhaltige Beeinträchtigung des sexuellen Empfindens herbeizuführen, kann nicht eingewilligt werden.

Dieser Passus richtet sich gegen die Praxis der Genitalverstümmelung (FGM), die in Österreich als schwere Körperverletzung nach $₫ 84.1$ StGB gilt. Paradox wirken die gleichzeitige Einführung strenger strafrechtlicher Bestimmungen gegen FGM bei Migrantinnen und die zunehmende Bereitschaft zur intimchirurgischen Experimentierfreudigkeit in Teilen der einheimischen Bevölkerung. Chirurgische Eingriffe am weiblichen Genital, die die WHO-Kriterien der FGM erfüllen würden, werden als Weiterentwicklung von Tätowierungen und Piercings erlebt, in die viele Frauen gerne einwilligen und auch bereit sind, teuer dafür zu bezahlen (Berer 2010). Bei Unzufriedenheit mit dem Ergebnis kann dann versucht werden, mit dem oben zitierten $₫ 90$ Abs. 3 des österreichischen StGB zu klagen [8]. Diese Entwicklung ist noch $\mathrm{zu}$ neu, als dass es orientierende Leitsätze der Rechtsprechung gibt. Es darf dabei keinesfalls übersehen werden, dass die ästhetische Chirurgie, die bis vor wenigen Jahren ganz den Kräften des medizinischen Marktes von Angebot und Nachfrage überlassen war, nun durch das Bundesgesetz über die Durchführung von ästhetischen Behandlungen und Operationen, ÄsthOPGesetz, neben der Organtransplantation und der assistierten Reproduktion der wohl am strengsten regulierte Bereich der Medizin in Österreich ist. Gerade GynäkologInnen sollten sich, bevor sie in diesem Bereich beratend oder chirurgisch tätig werden, genau über die sehr präzise ausformulierten gesetzlichen Bestimmungen und Erlässe, etwa zur Zeitachse zwischen Aufklärung und Eingriff und der verpflichtenden Vorhernachher-Dokumentation, informieren (http://www.aesthetischeoperationenaerzte.at/).
Fasst man zusammen, so zeigt sich, wie wichtig es gerade im Fach Gynäkologie und Geburtshilfe ist, aufmerksam die Neuerungen und Veränderungen mitzuverfolgen und das eigene medizinische Handeln und die Dokumentation desselben zu adaptieren. Endometriose wurde schon bei ägyptischen Mumien aus der Zeit der Pharaonen gefunden, die Krankheit hat sich nicht geändert, die Wahrnehmung und auch die Rechtsfolgen sind heute aber bereits anders, als sie es im Jahr 2000 waren. Der Niveausprung im geburtshilflichen Ultraschall war in wenigen Jahren möglich - man erwähnt nur ungern, dass dies eine Konsequenz einschlägiger Urteile und OGH-Erkenntnisse war und weniger dem spontanen Wunsch nach Qualitätsverbesserung entsprach. Naheliegend wäre, nach diesen Erfahrungen den gynäkologischen Ultraschall und seine Dokumentation rechtzeitig zu verbessern und nicht auf Gerichtsurteile zu warten. Die freihändige Ausgabe von Pillenmustern ohne gründliche Anamnese und Beratung hat völlig aufgehört, Spiralen sind zu den am besten dokumentierten Verhütungsmethoden geworden. Im Bereich der Intimchirurgie ist Zurückhaltung angebracht, bis Klarheit über die sehr widersprüchlichen Vorstellungen und Vorschriften in diesem Bereich herrscht. Keine Gynäkologin, kein Gynäkologe will Rechtsgeschichte schreiben und den eigenen Namen mit einem berühmten Höchstgerichtsfall für Jahre in allen Medizinrechtslehrbüchern und Jus-Studentenskripten verstichwortet sehen.

\section{Korrespondenzadresse}

\section{Ao. Univ.-Prof. Dr. Christoph Brezinka}

Department Frauenheilkunde, Universitätsklinik für Gynäkologie und Geburtshilfe, Medizinische Universität Innsbruck Anichstraße 35, 6020 Innsbruck, Österreich christoph.brezinka@i-med.ac.at

Funding. Open access funding provided by University of Innsbruck and Medical University of Innsbruck.

\section{Einhaltung ethischer Richtlinien}

Interessenkonflikt. C. Brezinka gibt an, dass kein Interessenkonflikt besteht.
Für diesen Beitrag wurden vom Autor keine Studien an Menschen oder Tieren durchgeführt. Für die aufgeführten Studien gelten die jeweils dort angegebenen ethischen Richtlinien.

Open Access. Dieser Artikel wird unter der Creative Commons Namensnennung 4.0 International Lizenz veröffentlicht, welche die Nutzung, Vervielfältigung, Bearbeitung, Verbreitung und Wiedergabe in jeglichem Medium und Format erlaubt, sofern Sie den/die ursprünglichen Autor(en) und die Quelle ordnungsgemäß nennen, einen Link zur Creative Commons Lizenz beifügen und angeben, ob Änderungen vorgenommen wurden.

Die in diesem Artikel enthaltenen Bilder und sonstiges Drittmaterial unterliegen ebenfalls der genannten Creative Commons Lizenz, sofern sich aus der Abbildungslegende nichts anderes ergibt. Sofern das betreffende Material nicht unter der genannten Creative Commons Lizenz steht und die betreffende Handlung nicht nach gesetzlichen Vorschriften erlaubt ist, ist für die oben aufgeführten Weiterverwendungen des Materials die Einwilligung des jeweiligen Rechteinhabers einzuholen.

Weitere Details zur Lizenz entnehmen Sie bitte der Lizenzinformation auf http://creativecommons.org/ licenses/by/4.0/deed.de.

\section{Literatur}

1. Nnoaham KE et al (2019) Reprint of: Impact of endometriosis on quality of life and work productivity: a multicenter study across ten countries. Fertil Steril 112(4 Suppl1):e137-e152

2. Taylor HS et al (2021) Endometriosis is a chronic systemic disease: clinical challenges and novel innovations. Lancet 397(10276):839-852

3. Garibaldi PD (2021) Endometriosi - il riconoscimento della sua rilevanza sociale è legge regionale. La Voce di Genova, 22 giugno

4. Krämer B (2020) Diagnostik und Therapie der Endometriose bei Kinderwunsch. Frauenheilkd Up2date 14:471-488

5. Müller C (2020) Kupferspiralen brechen. Dtsch Apoth Z. https://www.deutscheapotheker-zeitung.de/news/artikel/2020/01/23/ kupferspiralen-brechen.Zugegriffen:6.11.2021

6. Brezinka C (2021) Tipps und Tricks im GynUltraschall: Risikoberechnung von Ovarialzysten nach IOTA mit dem ADNEX-App. J Gyn Endokrinol. https://doi.org/10.1007/s41974-021-00176-w

7. Andreotti RF et al (2020) O-RADS US risk stratification and management system: $A$ consensus guideline from the ACR ovarian-Adnexal reporting and data system committee. Radiology 294(1):168-185

8. Brezinka C (2020) Gutachterliche Aspekte bei Eingriffen am weiblichen Genital-Genitalverstümmelung (FGM) vs Intimchirurgie. Ö Z Ärztl Gutacht 2:58-61

Hinweis des Verlags. Der Verlag bleibt in Hinblick auf geografische Zuordnungen und Gebietsbezeichnungen in veröffentlichten Karten und Instituts adressen neutral. 